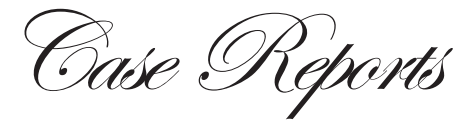

\section{Corrosive esophageal injury due to elemental sodium ingestion}

\section{Introduction}

Caustic ingestion can produce progressive and devastating injury to the esophagus and stomach. ${ }^{1}$ Although most patients survive the acute injury, the reparative process can result in esophageal and/or gastric stenosis and an increased risk of esophageal cancer. Button batteries contain high concentrations of sodium and potassium hydroxide and can cause severe injuries. Milder injuries are usually caused by less potent agents such as sodium carbonate, ammonium hydroxide and bleaches.

Although metallic sodium is known to produce esophageal burns, ${ }^{2-5}$ an extensive literature search failed to yield any case of esophageal burns produced by ingestion of elemental sodium. To the best of our knowledge, we describe here the first reported case of esophageal burns leading to stricture formation following ingestion of elemental sodium. We also review the relevant but scant literature on sodium metal ingestion.

\section{Case report}

A 42-year-old man, proprietor of an industrial testing laboratory swallowed a piece of sodium metal weighing about 10 grams. Handling of the metal resulted in burns over the palm and fingers, and the patient became unconscious for about 45 minutes. The patient on regaining consciousness complained of severe heartburn and retrosternal pain.There was oral regurgitation of serosanguinous slough for about 48 hours. The patient was unable to talk for 3 days. The incident occurred in a remote town and in the absence of any advice on the subject in available textbooks of medicine and toxicology, the treating physician empirically commenced treatment by administering esophageal and gastric lavage with potassium permanganate solution. The patient was also administered intravenous hydrocortisone which was continued for about 72 hours. In addition the patient was also administered intravenous antibiotics along with intermittent diclofenac for pain. The patient developed odynophagia, and after 19 days the patient was referred for an endoscopic examination.

Endoscopy revealed minimal swelling and scarring in the hypopharynx and confluent deep ulcerations with narrowing of lumen beginning right at the upper end of the esophagus. The endoscope could only be introduced up to $25 \mathrm{~cm}$ from the incisors. Visualization of middle and lower esophagus was not possible. The patient was then subjected to dilatation with Savary Gillard dilators at intervals of one to two weeks. After dilatation it was seen that the patient had narrowing and ulcerations upto the gastroesophageal junction.

During the subsequent 4 months, the patient underwent 17 sessions of dilatation. The last 2 sessions were supplemented by endoscopic corticosteroid injections into the esophagus after the dilatations. Following corticosteroid injections, the patient felt better; the intervals between the dilatation sessions lengthened from one week to a fortnight.

\section{Discussion}

Caustic ingestion is known to produce a progressive and devastating injury to the esophagus and stomach which can result in esophageal and/or gastric stenosis and an increased incidence of esophageal cancer. These caustic gastrointestinal injury are generally caused by an alkaline or acidic agent. ${ }^{1}$ Although a number of websites ${ }^{2-5}$ mention the potential adverse effects of sodium ingestion and the first aid measures which should be taken following metallic sodium ingestion, to the best of our knowledge, there is no published report in the English medical literature.

Sodium metal has an auto ignition temperature of $115^{\circ}, \mathrm{C}$, which suggests that it will not ignite at normal room temperatures. ${ }^{6}$ However, after the sodium is removed from its protective oil shroud, it is exposed to ambient air and moisture, which results in its oxidation external deposition of oxides and sodium hydroxide. These substances are hygroscopic and result in further accumulation of moisture and intensify the production of oxides and hydrogen gas, ultimately producing auto ignition at room temperature. Burns are the primary sequelae associated with exposure to elemental sodium. The burns are a consequence of the reaction of the elemental metal with the ambient moisture which produces a pronounced exothermic reaction and evolution of alkaline metallic hydroxide. Thus elemental sodium produces a combination of chemical and thermal injury. ${ }^{7}$

Two industrial material safety websites in their warning describe the potential health effects as follows. 'Sodium metal 
is an extremely dangerous, corrosive material. On ingestion this will react immediately with saliva to cause serious burns and possible local combustion and even explosion of hydrogen in the mouth or esophagus. The metal's low melting point can cause further complications'.2,4 They also issue clear instructions regarding the immediate first aid measures to be adopted. All the websites give special emphasis tothe warning: 'Do not induce vomiting'. If the victim is conscious and alert, they advocate administration of large quantities of milk or water. Besides, they uniformly exhort not to give anything by mouth if the person is unconscious. ${ }^{2-5}$ However, despite these descriptions regarding the toxic effects of sodium and the first aid measures needed, we could not find a single case report on the subject. Unfortunately in the present case, in the absence of any published literature on the subject, the patient was subjected to induced vomiting by the treating physician.

As there were extensive deep burns with complete mucosal denudation and stricture formation, the patient needed dilatation sessions with Savary Gillard dilators every week in order to maintain nutrition. After 15 sessions, he was administered corticosteroid injections into the esophagus over multiple sites along the entire length. Following corticosteroid therapy, the patient felt better and the intervals between the dilatation sessions lengthened from one week to a fortnight. We plan to insert an expandable plastic stent after a few more dilatations, after the upper and lower ends of the esophagus become healthier with restoration of the mucosa.

This case of corrosive esophageal injury due to elemental sodium ingestion has been reported because of its rarity, with the hope that in future, textbooks of toxicology would make some mention of the measures to be adopted in a case of elemental sodium poisoning.

\section{SHIVARAM PRASAD SINGH DEBASIS MISRA ${ }^{1}$ \\ MANAS KUMAR PANIGRAHI ${ }^{1}$ INDU BHUSAN KAR ${ }^{2}$ OMPRAKASH AGRAWAL ${ }^{3}$ CHUDAMANI MEHER ${ }^{3}$}

Correspondence: Dr. Debasis Misra Departments of Gastroenterology $y^{l}$ and Oral and Maxillofacial Surgery, ${ }^{2}$ S.C.B. Medical College, Cuttack 753007, Odisha, India Department of Radiology, ${ }^{3}$ Beam Diagnostics Center, Cuttack 753001, Orissa, India Email: dmisra.75@gmail.com

\section{References}

1. Loeb PM, Nunez MJ. Caustic injury to the upper gastrointestinal tract. In: Feldman M, Friedman LS, Sleisenger MH, editors. Sleisenger and Fordtran'sgastrointestinal and liver disease: pathophysiology, diagnosis, management. 7th ed. Philadelphia: W.B. Saunders Co; 2002.p. 399-407.

2. Material safety data sheet. Sodium metal. Available from: http:/ /www.jtbaker.com/msds/englishhtml/s2594.htm

3. Material safety data sheet: sodium metal. Available from: http:// avogadro.chem.iastate.edu/MSDS/Na.htm

4. Materials safety data sheet - sodium metal. Available from: http:/ /www.rct.edu.sa/chm/safety/msds/sodium.pdf

5. Material safety data sheet: sodium metal. Available from: http:// www.matheson-trigas.com/msds/MAT20850.pdf

6. Meyer E. Chemistry of hazardous materials. Engleweed Cliffs, NJ: Prentice Hall; 1977.p.156-161.

7. Clare RA, Krenzelok EP. Chemical burns secondary to elemental metal exposure: two case reports. Am J Emerg Med. 1988;6:355-7.

\section{A rare case of atypical hemangioma showing centrifugal enhancement}

\section{Introduction}

Hepatic hemangiomas are the most common benign hepatic masses with a prevalence of $1-20 \%{ }^{1}$ They are more commonly seen in females than males with a ratio varying from $2: 1$ to $5: 1{ }^{1}$ Typical radiographic characteristics of hepatic hemangiomas are well described and extremely specific. However, intratumoral structural variation may cause unusual imaging features. This can lead to misleading diagnosis. Hence, knowledge of imaging features of atypical hemangiomas is imperative to avoid unnecessary interventions.

\section{Case report}

A 46year-old female patient presented with history of pain in right hypochondrium since four months. The pain was associated with two episodes of vomiting over last three days. There was no history of fever. On examination, mild hepatomegaly was noted with tenderness in right 